Controlling microtubule acetylation

Nachury and colleagues (Dev. Cell 15, 854-865; 2008) describe a protein named BBIP10 that regulates microtubule acetylation and polymerization. Previously, the authors had identified the BBSome complex, which is important for ciliary membrane trafficking and contains proteins encoded by genes mutated in BardetBiedl Syndrome (BBS). They found that BBIP10 is essential for ciliogenesis in cultured cells and in zebrafish, and its depletion specifically affected BBSome assembly. Although BBIP10 is a $\mathrm{BBSome-associated} \mathrm{protein,} \mathrm{a} \mathrm{significant}$ fraction of BBIP10 was also found to be nonBBSome-associated. Remarkably, BBIP10 depletion reduced cytoplasmic tubulin acetylation and microtubule density, phenotypes not seen when other BBSome components were depleted, suggesting independent effects of BBIP10 on tubulin acetylation and ciliogenesis. In vitro, BBIP10 associated with microtubules and facilitated the formation of microtubule asters from purified tubulin. However, in cells, BBIP10 seemed to influence tubulin acetylation independently of the effects on microtubule polymerization. Furthermore, the authors found that depleting the tubulin deacetylase Hdac6 restored tubulin acetylation levels in BBIP10-depleted cells. As BBIP10 also interacts with Hdac6, it may affect tubulin acetylation by acting on Hdac6 but the details remain to be determined.

SS

\section{Desumoylation controls trophoblast development}

Conjugation of the ubiquitin-related moiety SUMO to protein substrates has been implicated in a wide variety of cellular functions. Hsu and colleagues (Plos Biol. 6, e310; 2008) now provide further support for the physiological importance of sumoylation by showing that SUMO-specific protease 2 (SENP2), member of a SUMO protease family of proteins that remove SUMO from substrates, is essential for trophoblast development. Trophoblast development depends on the expansion of progenitor cells, followed by differentiation into three main lineages (labyrinth, spongiotrophoblast and polyploid trophoblast giant cells (TGCs)), which requires endoreduplication. Mouse embryos lacking SENP2 had defects in both progenitor and differentiated trophoblasts. SENP2-null cells underwent cell-cycle arrest specifically at the G1-S cell-cycle transition, thus altering both mitosis and endoreduplication. Cellcycle arrest was caused by accumulation of the tumour suppressor p53, as p53 silencing rescued cell-cycle progression and TGC differentiation. The E3 ubiquitin ligase $\mathrm{Mdm} 2$ regulates p53 levels by inducing its degradation and is known to be modified by SUMO. Indeed, SENP2-null cells aberrantly accumulated sumoylated Mdm2 in the nucleus, resulting in p53 stabilization. Re-expression

\title{
A pore to escape
}

Perforins secreted by bacteria or cells of the mammalian immune system induce death of target cells through their pore-forming activity, but the mode of action of perforin-like proteins (PLP), present in a number of pathogens, has been unclear. Carruthers and colleagues (Science doi10.126/science.1165740) have found that Toxoplasma gondii PLP1, the amino acid sequence of which has features characteristic of pore proteins, is essential for parasite egression from the cytoplasm of host cells. Toxoplasma depends for its exit on the activity of a secretion apparatus known as the microneme. PLP1 localizes to the microneme, and is secreted after calcium stimulation. In cell culture, the exit of parasites carrying a deletion of the $p l p 1$ gene was delayed and, under these conditions, membranes frequently surrounded those that egressed. The authors observed that permeabilization of the vacuole that encases the parasite in the cytoplasm was rarely seen in cells infected with Toxoplasma lacking plp1, but this defect could be rescued by co-infection with wild-type parasites. These data indicate that PLP1 is essential for membrane permeabilization before Toxoplasma exit from cells. Mice infected with ten or more parasites usually died within fifteen days, whereas mice infected with up to a million of Toxoplasma lacking plp1 stayed healthy. It remains to be investigated whether the malaria parasite, Plasmodium falciparum, which belongs to the same phylum as Toxoplasma, also uses PLPs to egress from erythrocytes. NLB

of SENP2 reduced Mdm2 sumoylation, decreasing p53 levels and promoting TGC differentiation. Although it will be important to clarify whether Mdm2 is the only and direct target of SENP2 in this context, the role of SENP2 in regulating the Mdm2-p53 pathway may cast light on the mechanisms that prevent polyploidy in normal and pathological cell growth.

SG

\section{Heparan sulphate code directs neurons}

In the ventral nerve cord of the Caenorhabditis elegans embryo, axons of DA and DB motor neurons extend either along the left or the right side of the animal. How axons choose their direction is explained in a study by Hobert and colleagues (Curr. Biol. 18, 19781989; 2008). Different heparan sulphate (HS) modifications on proteins have been found to be essential for correct neuronal patterning, but whether they have an instructive or permissive guidance role is unclear. Hobert and colleagues found that genetic removal of three types of HS modification in different combinations caused distinct defects in the routing of individual motor neurons. Furthermore, aberrant expression of the HS-modifying enzyme, 6-O-sulphotransferase, in the hypodermis caused misrouting of neuronal projections, showing that the HS-modification pattern specifies axonal direction. Genetic analysis suggested that HS modification on the HS core protein lon-2/glypican mediates misrouting of a specific motor neuron, DB7. The axon-guidance cue slt-1/Slit is known to require HS modifications in various systems, and the authors found that misrouting of DB7 required the presence of slt- 1 and its receptor eva-1. Although the findings need to be corroborated biochemically, they suggest that regional HS-modifications have an instructive role in axon guidance, possibly by determining the responsiveness of neurons to ligand/receptor patterning cues. CKR

Written by Silvia Grisendi, Nathalie Le Bot, Christina Karlsson Rosenthal and Sowmya Swaminathan 